\title{
Biofeedback Intervention for Anger Management: A Case Study
}

\author{
Charles J. Chapman
}

Eastern Washington University

\begin{abstract}
Stress is usually tied into anger, both of which have been shown to have negative health effects. Biofeedback has been shown to reduce stress (which can be anger-inducing) and has been suggested as an intervention. This study will determine the effects of biofeedback on anger management by monitoring heart rate variability (HRV) through focused breathing and positive thoughts (such as that of a loved one). The settings were at home and in public. The baseline was 12 days; interventions occurred twice daily, with times of each increasing every 7 days and tracked for 4 consecutive weeks. Consistently applied, the intervention indicated a significant reduction in the number of anger events as well as a marked decrease in the intensity of each event.

Keywords: anger; biofeedback; intensity; intervention; stress

Citation: Chapman, C. J. (2017). Biofeedback intervention for anger management: A case study. NeuroRegulation, 4(2), 95-98. http://dx.doi.org/10.15540/nr.4.2.95

*Address correspondence to: Charles J. Chapman, Eastern Washington University, 2003 E. Columbia Avenue, Spokane, WA 99208, USA. Email: charlesjchapman63@yahoo.com

Edited by:

Rex L. Cannon, PhD, Knoxville Neurofeedback Group, Knoxville, Tennessee, USA

Copyright: (c) 2017. Chapman. This is an Open Access article distributed under the terms of the Creative Commons Attribution License (CC-BY).

\section{Reviewed by:}

Rex L. Cannon, PhD, Knoxville Neurofeedback Group, Knoxville, Tennessee, USA

Randall Lyle, PhD, Mount Mercy University, Cedar Rapids, lowa, USA
\end{abstract}

\section{Introduction}

Many studies discuss biofeedback and its effect on stress relief as well as other health conditions (Greenspoon \& Olson, 1986; Shellenberger, Turner, Green, \& Cooney, 1986; Wyner, 2015), yet few appear to solely address the effects of biofeedback on anger as its own construct. One of those studies, conducted in the United Kingdom, determined that self-intervention, along with self-monitoring, was effective in treating anger management versus selfmonitoring alone (Fernandez \& Beck, 2001). Another study suggests that heart rate variability (HRV) can be manipulated to address the response to stimuli that produce anger. Although not conclusive, the results did provide support for the use of biofeedback as a method of tracking and regulating emotions (Francis, Penglis, \& McDonald, 2016).

Anger is prevalent in our society, and crimes involving anger add to an already overburdened justice system. Anger is costly in terms of lost wages/jobs, medical care for victims, friendships lost, and families broken. This is especially true when anger manifests as domestic violence. For example, the Spokane (WA) County Regional Health District, citing data provided by the Washington Association of Sheriff and Police Chiefs, reports the rate of Spokane County domestic violence offenses reported to law enforcement in 2013 as 8.7 per 1,000 population-an almost $50 \%$ increase above the statewide rate of 5.9 per 1,000 population in the same time frame. Spokane Cares, citing a report from the Washington State Coalition Against Domestic Violence, states that Spokane County had two domestic-violence-related homicides and two suicides in 2013; in 2014, the numbers increased to four and two, respectively. In 2015, Spokane County had eight domestic-violence-related homicides and one suicide.

This particular case study, an assignment for a university Behavior Modification course, is intended to determine what effects, if any, biofeedback has on anger management. It is not designed to address stress relief for two reasons: (1) subject reports 
becoming angry even when there is no noticeable stress, and (2) it is assumed that any stress levels that exist will lessen should the anger decrease.

Medically defined, anger is:

An emotional reaction characterized by extreme displeasure, rage, indignation, or hostility. It is considered to be of pathological origin when such a response does not realistically reflect a person's actual circumstances. However, expressions of anger vary widely in different individuals and cultures and may be considered functional under certain controlled circumstances (Mosby's, 2009).

In 2008, the Association for Applied Psychophysiology and Biofeedback (AAPB), the Biofeedback Certification International Alliance (BCIA), and the International Society for Neurofeedback and Research (ISNR) agreed upon a working definition for biofeedback:

A process that enables an individual to learn how to change physiological activity for the purposes of improving health and performance. Precise instruments measure physiological activity such as brain waves, heart function, breathing, muscle activity, and skin temperature. These instruments rapidly and accurately "feed back" information to the user. The presentation of this information-often in conjunction with changes in thinking, emotion, and behavior-supports desired physiological changes. Over time, these changes can endure without the use of an instrument (AAPB, 2008).

\section{Method}

\section{Participant}

The subject, a 54-year-old male, indicates lifelong, high-intensity anger issues and extreme difficulty controlling his temper. He reports daily physical, emotional, and verbal abuse throughout childhood by the male parent. Attempts in childhood and early adulthood to address the condition yielded no results. Anger arousal and expression have cost him jobs and relationships and caused issues with campus personnel. Previous to the biofeedback intervention, he has not been effective in the implementation of anger management tools he has learned, as his anger escalates immediately upon a stressor being presented; the hypothesis is that biofeedback will allow him to have time to use the techniques he has learned by beginning at a lower intensity level, resulting in not getting as angry as quickly.

Of his own volition, subject has been regularly attending anger management counseling for over one year and has recently been introduced to biofeedback as a possible intervention.

\section{Apparatus}

In addition to ongoing counseling, the emWave2 portable biofeedback unit from HeartMath, Inc. (Boulder Creek, CA) was utilized. According to one of HeartMath's instructional flyers, the object is to create "coherence," which they define as "a state of synchronization between your heart, brain, and autonomic nervous system...achieved and sustained by activating a positive feeling. It is not the same as relaxation, rather reflects a state of relaxation and revitalization at the same time (being in an optimal state of efficiency)." Readouts are lighted electronic display (LED), obtained by attaching the earclip electrode to the ear and plugging the other end into the unit. Upon activation, the electrode reads the subject's heart rate and displays the appropriate level of coherence as either a red, blue, or green LED.

\section{Design and Procedure}

Subject defines anger events as behavior resulting from stimuli that produce a negative verbal and/or physical reaction and that negatively affect mood for more than two to three minutes. The design is across settings (at home and in public). Frequency and intensity are measured, with intensity being reported on a scale created by the subject of 0 (not angry) to 10 (homicidal). Thirty-two anger events occurred during the 12-day baseline $(M=2.6667$, where $N=12, \mathrm{p}=29, \mathrm{~h}=3 ; N=$ total number of days, $p=$ in public, and $h=$ home).

The base intensity at home was usually a 1 ; in public, usually a 2 (subject states that just being out in public increases his base intensity level). Reinforcement behavior included replacing angry thoughts with thoughts of his fiancée.

In addition to ongoing counseling, and with few exceptions where noted, subject followed the HeartMath protocol for Quick Coherence. Beginning by focusing on his heart, subject then imagined himself breathing through it, slower and deeper than normal. Interventions were twice daily, beginning at 2-3 min each, with additional interventions as required. Subject chose 7 -day intervals between 
increasing intervention times, with an 8-day period coinciding with month's end in February. After 7 days, times increased, first to 5-6 min, then to 7-8 min, and finally to $10-11$ min. During that 4-week period, there were 7 nonconsecutive days with only one intervention. There are four challenge levels on the unit; subject performed the intervention using the lowest level.

The graph below shows both mean frequency (in blue) and mean intensity (in red) for anger events occurring during the baseline and for each week of intervention over the course of the study. For convenience, these numbers have been rounded up or down accordingly prior to creation of the graph.

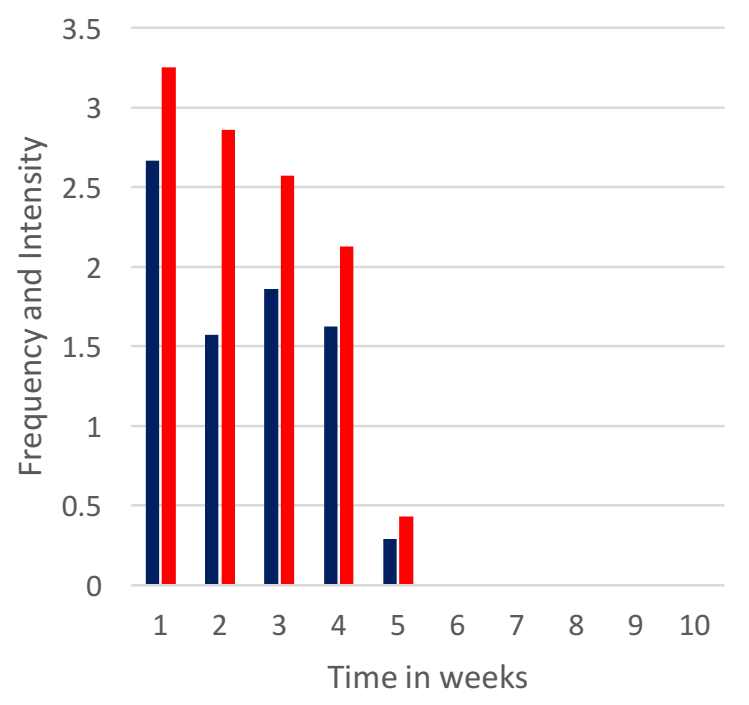

Figure 1. Mean frequency and mean intensity for anger events occurring during the baseline and for each week of intervention.

\section{Results}

During the first week of intervention, 10 anger events occurred ( $M=1.4286 ; N=7, p=8, h=2)$; intensity $M=3$. The second week saw a slight increase with 13 anger events occurring $(M=$ 1.8751; $N=7, \mathrm{p}=12, \mathrm{~h}=1)$; intensity $M=3$. The third week ( 8 days) the anger events leveled off at $13(M=1.625 ; N=8, \mathrm{p}=13, \mathrm{~h}=0)$; the intensity dropped $(M=2.5333)$. During the last 7 days of intervention, there were only two anger events $(M=$ $0.2857 ; N=7, p=2, h=0)$; and the intensity was reduced even further $(M=0.6667)$.
No anger events occurred on 2 days during the 12 day baseline, equating to $16.667 \%$ of the time $(N=$ total number of days, $n=$ number of 0 anger event days). During the first and second 7-day periods of intervention, there were 2 days each with no anger events, or $28.57 \%$ of the time ( $N=7, n=2$ for each). The third week of intervention ( 8 days) had 3 days with no anger events, or $37.5 \%$ of the time $(N=8, n$ $=3$ ). The last 7 days of intervention had the most days with no anger events, 6 days, or $85.7 \%$ of the time $(N=7, n=6)$. During the 29 days of intervention, there were 13 days with no anger events, or $44.83 \%$ of the time $(N=29, n=13)$.

\section{Discussion}

The results of this case study coincide with the results of Fernandez and Beck's study (2001) and appear to support the conclusions reached by Francis, Penglis, and McDonald (2016), both mentioned at the beginning of this case study. The results support the hypothesis presented earlierthat the consistent use of biofeedback as an intervention can be a beneficial tool for the management of anger. The results imply that biofeedback intervention can also be useful in reducing the number and severity of domestic violence cases as well as other anger-related criminal offenses.

The dramatic drop in the number of anger events and the decrease in the intensity of each event is unexpected, given the short amount of time to date; much of this improvement is attributed to the consistent application of the intervention. It is highly doubtful that these results would be seen without duly applying the intervention. Of his own accord the subject has agreed to the continuation of this study until the equipment must be returned midJune. Given the results experienced to date, he is eager to see how successful the intervention will be at 60 and 90 days. Bearing in mind that the results will vary from individual to individual, further research on biofeedback's effectiveness as an intervention for anger management is highly encouraged. The limitations of this case study are twofold: a single participant and self-administered.

\section{Author Note}

A few days prior to the submission of this revision, the author's mother passed away. This paper and the results experienced are dedicated to her memory. 


\section{References}

Anger. (n.d.). In Mosby's Medical Dictionary. (2009). (8th ed.). St. Louis, MO: Mosby Elsevier. Retrieved February 28, 2017, from http://medical-dictionary.thefreedictionary.com/anger

Association for Applied Psychophysiology and Biofeedback. (2008, May 18). What is Biofeedback? Retrieved from http://www.aapb.org

emWave2 system [Apparatus]. Boulder Creek, CO: HeartMath, Inc. https://store.heartmath.com/emwave2

Fernandez, E., \& Beck, R. (2001). Cognitive-behavioral selfintervention versus self-monitoring of anger: Effects on anger frequency, duration, and intensity. Behavioural and Cognitive Psychotherapy, 29(3), 345-356. http://dx.doi.org/10.1017/S1352465801003071

Francis, H. M., Penglis, K. M., \& McDonald, S. (2016). Manipulation of heart rate variability can modify response to anger-inducing stimuli. Social Neuroscience, 11(5), 545-552. http://dx.doi.org/10.1080/17470919.2015.1115777

Greenspoon, J., \& Olson, J. (1986). Stress management and biofeedback. Clinical Biofeedback and Health: An International Journal, 9(2), 65-80.
Shellenberger, R. D., Turner, J., Green, J., \& Cooney, J. B. (1986). Health changes in a biofeedback and stress management program. Clinical Biofeedback and Health: An International Journal, 9(1), 23-34.

SpokaneCares.org. (n.d.). Domestic violence: Statistics. Retrieved from http://www.spokanecares.org/domesticviolence-spokane.php - stats

Spokane Regional Health District. (n.d.). Spokane Counts 2015: Domestic Violence. Retrieved from http://www.srhd.org /spokanecounts/indicator/46/domestic-violence

Wyner, D. R. (2015). Pilot study of a university counseling center stress management program employing mindfulness and compassion-based relaxation training with biofeedback. Biofeedback, 43(3), 121-128. http://doi.org/10.5298/10815937-43.3.01

Received: May 19, 2017

Accepted: June 21, 2017

Published: June 30, 2017 